\title{
A Comparative Study of Bond Order and Bond Length Calculations of Some Conjugated Hydrocarbons using Two Different Methods
}

\author{
Manal O. Hamzah \\ Department of chemistry, college of science of women, University of Baghdad, Jadriah, Baghdad, \\ Iraq
}

\begin{abstract}
In the present work, bond lengths and bond orders of a selected set of benzenoid molecules were calculated. The calculations of bond orders were carried out using two different approaches, Pauling and Coulson. The two versions of Pauling equation of bond length that predicted in 1945 and 1960 were used to calculate the bond lengths. A new set of parameters called $R_{\text {new }}$ was used for calculating the bond lengths. The ratio of force constant $F$ in Pauling equation of bond length of the set of molecules was also calculated. At each value of $F(0.05,0.10,1.5,2.0,2.5,3.0,3.5,4.0,4.5,5.0,10.00$, and 0.001), 101 values of Pauling bond orders PBOs and relative bond lengths $R$ were calculated. to determine the best value of $F$. Then the results were compared.
\end{abstract}

Keywords: bond order, bond length, benzenoid, Pauling, Coulson, Kekuléan.

\section{INTRODUCTION}

One of the most significant discussions in chemistry is the bond length and bond order calculations. During the 1930s, many attempts by Pauling [1], Lennard Jones [2], Penney [3], Coulson [4] and others were carried out to define the bond order. The first attempt to define the bond order was introduced by Pauling, Brockway and Beach [1] in 1935. It was based on valence bond theory (VBT) [4].Pauling bond order (PBO) is now the name associated with this definition. Their assumption was that amolecule in the ground state has a wave function represented by a series of canonical structures [4] or Kekule structures $(K)$. It was also assumed that in a benzenoid the contribution of each of these structures to the $\pi$-electron system of carbon-carbon bonds is equal [5]. The bonds in Kekule structures are specified either as formally double or single. Hence, suitable summation over structures enables a fractional bond order to be defined [4].

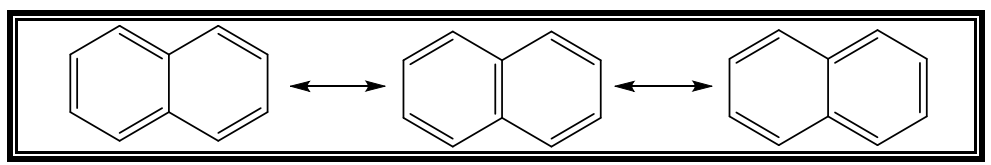

Figure1. Kekulé structures of naphthalene

In a conjugated molecule, PBO was defined as a ratio between two numbers related to these structures [5].

$P_{i j}^{P}=\frac{K_{i j}}{K}$

where $P_{i j}^{P}$ refers to Pauling bond order, $K$ is the total number of Kekulé structures in a conjugated molecule, and $K_{i j}$ is the number of Kekule structures in which the particular chemical bond (ij) exists as a double bond. The formula (1) applies to conjugated systems containing single and double bonds. For $\pi$-conjugated systems containing single, double, and triple bonds, the PBO is given as [6]

$P_{i j}^{P}=\frac{1}{K} \sum_{K=1}^{K_{i j}} n_{i j}(k)$

Where

$n_{i j}(k)=\left\{\begin{array}{c}1 \text { for a double bond } \\ 2 \text { for a triple bond }\end{array}\right\}$ 
The PBOs according to formulas (1) and (2) give weights of the $\pi$ bondon the chemical bond (ij), which are measures of chemical bond (ij) strength [6].Counting the Kekule structures of benzenoid hydrocarbons have been described in a large volume of published studies [7]. For single chain condensed aromatic molecules, Cyvin [8] derived some equations to calculate the Kekule structures of linear chains (polyacenes such as naphthalene) and bent chains (polyphenes such as tetraphene) respectively. The counts are

$K=Q+1$

$K=\left\{\begin{array}{ll}1+\frac{1}{4}(Q+1)^{2} & Q=1,3,5, \ldots \\ 1+\frac{1}{4} Q(Q+2) & Q=2,4,6, \ldots\end{array}\right\}$

Where $Q$ isthe number of benzene rings

Another approach of bond order calculations was in 1939, when Coulson derived a definition of bond order based on the molecular orbital theory (MOT) that said "the electrons are allocated to orbitals which spread over the molecule as a whole, subsequently the sharing and delocalization of the electrons are emphasized', [9].The Coulson bond order CBO was calculated using equation (6).

$P_{i j}^{C}=\sum_{n} g_{n} c_{i}^{n} c_{j}^{n}$

Where $g_{n}$ is the occupation number of the $n$th molecular orbital, $c_{n}$ are the eigenvectors of the adjacency matrix $M_{i j}$ which they defined as [10]

$$
c_{n}=\left[\begin{array}{c}
c_{i}^{n} \\
\cdot \\
\cdot \\
\cdot \\
c_{N}^{n}
\end{array}\right]
$$

Where $N$ is the number of electrons in occupied orbitals.

$M_{i j}=\left\{\begin{array}{ll}1 & \text { if } i, j \text { are neighbours } \\ 0 & \text { otherwise }\end{array}\right\}$

Both definitions of bond order, Pauling and Coulson were used successfully to predict the bond lengths of conjugated systems. In 1930s, Pauling suggested a correlation between bond lengths and bond orders. This empirical correlation included four points with bond orders and bond lengths asethene $(1,1.34 \AA)$, benzene $(0.5,1.38 \AA)$, graphite $(0.75,1.42 \AA)$, and ethane $(0,1.54 \AA)$. In 1948 , the equation that calculating bond lengths which are corresponded to Pauling bond orders was described as [11]

$R=R_{1}-\left(R_{1}-R_{2}\right) \frac{F P_{i j}^{P}}{(F-1) P_{i j}^{P}+1}$

Where $R_{1}$ is the single bond distance and was equal to $1.540 \AA, R_{2}$ is the double bond distance and was equal to $1.330 \AA$ and $F$ is the ratio of force constant which was equal to 3 . In 1960, the $F$ value was justified to 1.84 and $R_{l}$ and $R_{2}$ equal to 1.504 and $1.334 \AA$, respectively. This modification has been given a bond length-bond order correlation with only slight errors from the measured points [5].The method of Pauling was as effective as molecular orbital (MO) method to predict bond lengths of particular benzenoid hydrocarbons (naphthalene, anthracene, chrysene, quaterrylene and 1,2,8,9dibenzacridine) [12].

Coulson described the equation of calculation of bond lengths as

$R=s-\frac{s-d}{1+\frac{f_{s}}{f_{d}}\left(\frac{1-P_{i j}^{C}}{P_{i j}^{C}}\right)}$

Where $s$ and $d$ are the lengths of pure single and double bond, $f_{s}$ and $f_{d}$ are the corresponding force constants [12] and are equal to $2.48 \times 10^{5}$ and $4.90 \times 10^{5} \mathrm{dynes} / \mathrm{cm}$, respectively. This correlation was applied to predict the bond lengths in conjugated molecules [4].

In the present work, for a selected set of benzenoids, the calculations of bond lengths and bond orders 
were carried out using definitions of Pauling and Coulson bond order. This set of polycyclic aromatic hydrocarbons was selected because it considers a good source of data of electronic structure [13].

\section{MeThodology}

The bond lengths and bond orders calculations of the set of molecules were carried out using two models. This set consists of 27 benzenoids with 309 unique bonds, Fig.2.Many programs were used to predict the bond lengths and bond orders. These programs were written using FORTRAN 77 programming language [14]. Most of these programs were written as scripts (high level language) such as doedge, that plots the molecules with the numbers of edges; doletter, that plots the edges of molecules with letter; do Draw Pauling R, that plots the edges of molecules with the predicted Pauling bond lengths and dotranslate, that plots the edges of molecules with experimental and predicted Pauling bond lengths.

a) The Optimal Value of the Ratio of Force Constant F: to determine the optimal value of Fin equation (9) at different $F(0.05,0.10,1.5,2.0,2.5,3.0,3.5,4.0,4.5,5.0$, and 10.00). First, the parameters $F, R_{1}$ and $R_{2}$ were chosen as $1.84,1.54 \AA$ and $1.33 \AA$. Then, at each $F, 101$ values of bond lengths $R$ and Pauling bond orders PBOs were calculated. Finally, in addition to the experimental correlation of PBOs and $R$ of Pauling bond orders-bond lengths in 1935, the predicted PBOs and $R$ were correlated.

b) The Bond Lengths Calculations: the bond lengths of the set of molecules were predicted using the Pauling equation in 1948 with parameters as $\left(R_{1}=1.54 \AA, R_{2}=1.33 \AA\right.$ and $\left.F=3\right)$, the Pauling equation in 1960 with parameters as $\left(R_{1}=1.504 \AA, R_{2}=1.334 \AA\right.$ and $\left.F=1.84\right)$ and the Pauling equation with new parameters as $\left(R_{1}=1.54 \AA, R_{2}=1.33 \AA\right.$ and $\left.F=1.84\right)$. These equations are called as $R_{1948}, R_{1960}$ and $R_{\text {new }}$, respectively. Finally, theoretical bond lengths $R_{\text {theo, }}$ of molecules were calculated using Gaussian 09.

c) The Bond Order Calculations: Pauling bond orders PBOs were predicted using eq. (1).The Coulson bond orders CBOs of the set of benzenoids were calculated using equation (6) and those orders of non Kekuléan conjugated hydrocarbons were calculate using the simple Hückel molecular orbital SHMO program. The Microsoft office excel was used to correlate the results and to explore the connections between bond lengths and bond orders.

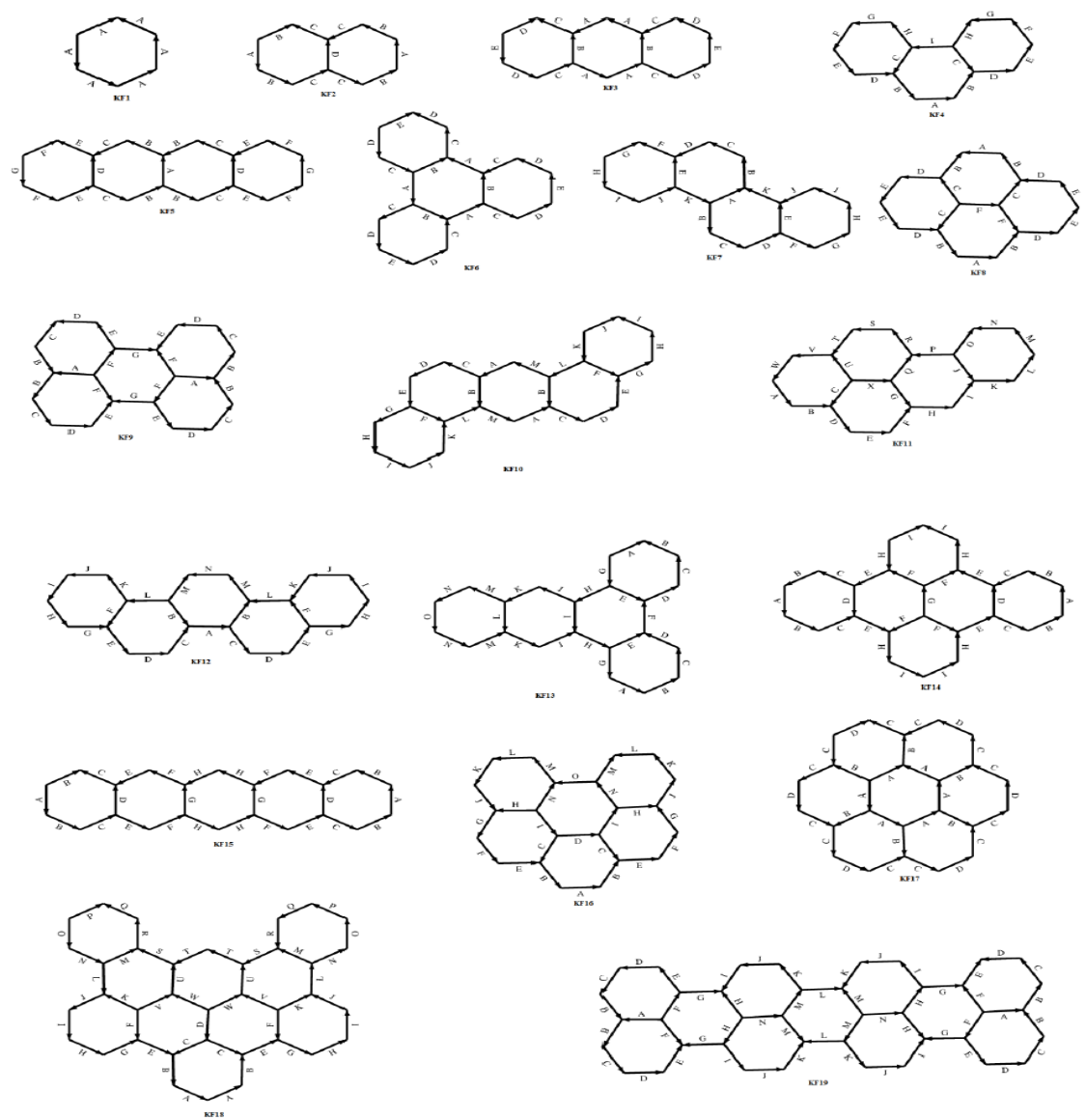




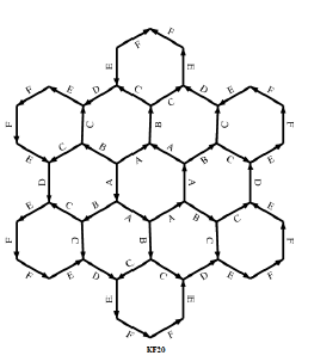

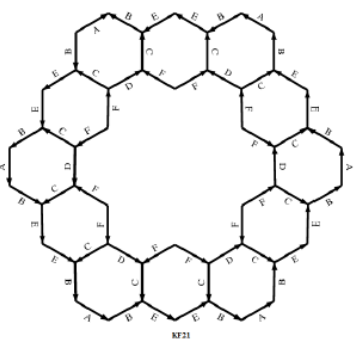

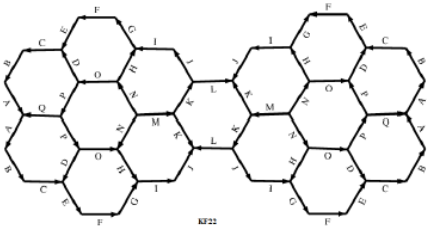<smiles>C1CCC2CC3C(CCC4CCCCC43)CC2C1</smiles><smiles>C1CCC2C(C1)CC1CC3C4CCCCC4C4CCCCC4C4CCCC1C4C23</smiles>

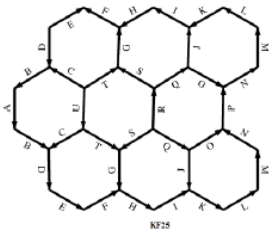

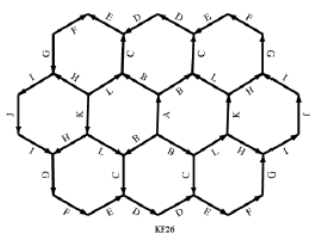

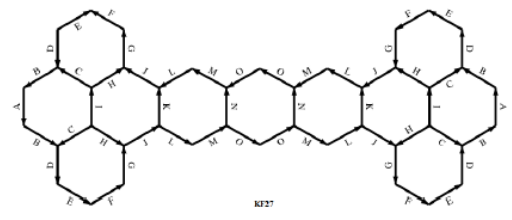

Figure2. The geometric molecular structures of the set of benzenoids

\section{RESULTS}

a) The Optimal Force Constant F: At each value of $F(0.05,0.10,1.5,2.0,2.5,3.0,3.5,4.0,4.5$, 5.0, 10.00, and 0.001), 101 values of Pauling bond orders PBOs and relative bond lengths $R$ were calculated. The calculated PBOs and $R$ were correlated. The correlations have shown that the deviation from the experimental correlation (ethylene, benzene, graphite, and ethane) was too big at $F$ equal to 0.001 and it was too small at $F$ equal to 10.00 , but it was identical to experimental correlation when $F$ is equal to $2.5 \mathrm{Fig}$. 3. This result indicates that the new $F$ of 101 calculated PBOs and $R$ was intermediate between 3 and 1.84, the $F$ of Pauling equation in 1948 and 1960, respectively

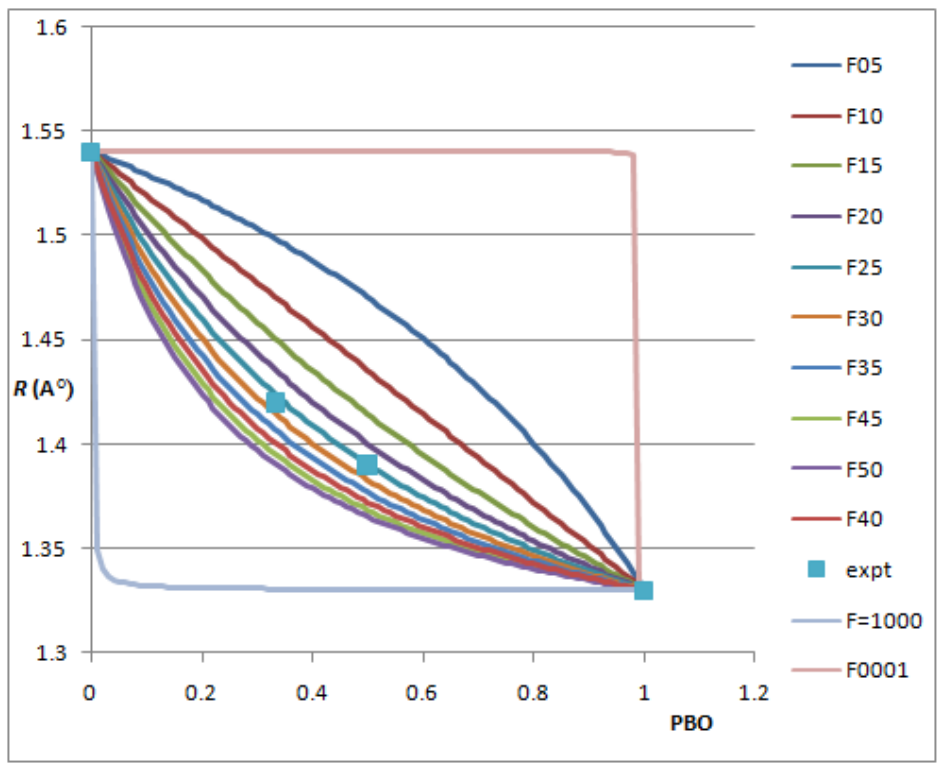

Figure3. The correlation between predicted Pauling bond orders PBO and bond lengths $R$ at different force constant $F$

b) The Calculations of Bond Lengths: the bond lengths of the set of molecules were calculated using Pauling equation in 1948, 1960 and new. The calculated lengths are listed in table (1). This table included the set of molecules with their symbols. These symbols refer to the first authors names of the journal article that provided the data of symmetry and measured bond lengths of the unique bonds (unshifted with symmetry operations). In addition it shows the predicted Pauling and Coulson bond orders. 
Many correlations were drawn. Figure 4 showed the correlation between $R_{1948}$ and $R_{1960}$. As shown, the correlation was linearly increased ( $R^{2}$ equal to 0.9824$)$. This value of $R^{2}$ indicated that the two equations in 1948 and 1960 were good to predict $R$ for the set of benzenoids.

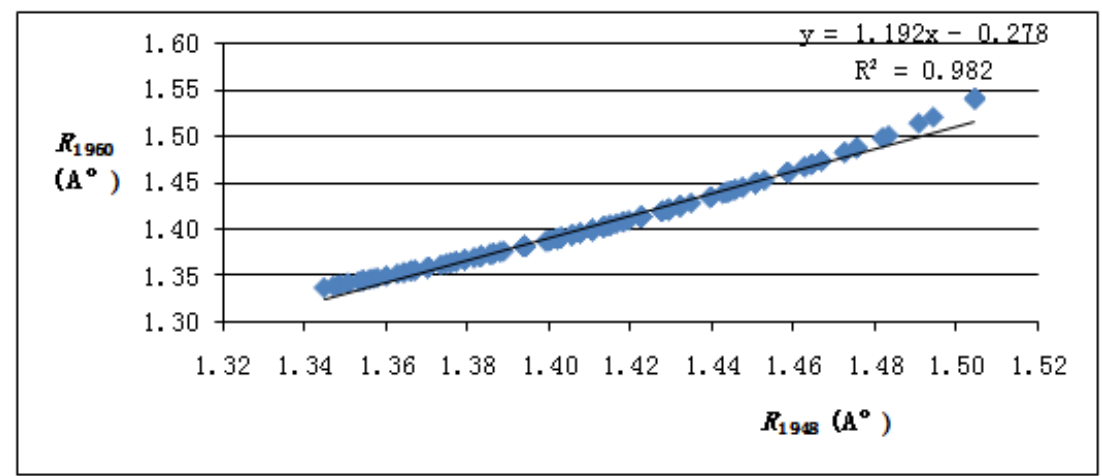

Figure4. The correlation between predicted lengths in 1948 and predicted lengths in 1960

Then, the predicted bond lengths $\left(R_{1948} R_{1960}\right.$ and $\left.R_{\text {new }}\right)$ were correlated with experimental $R_{\exp }$ to investigate the closeness between predicted and experimental values. As shown in Fig. 5, these correlations were linear ( $R^{2}$ equal to 0.7477 ) in 1948and (equal to 0.7783) in 1960and $R_{\text {new }}$. These values of $R^{2}$ indicated that the $R_{1960}$ and $R_{\text {new }}$ were identical and slightly closer to $R_{\exp }$ than $R_{1948}$. Thus, it could conclude that the Pauling equation with the sets of parameters in 1960 and new are more efficient than that equation with the set in 1948.

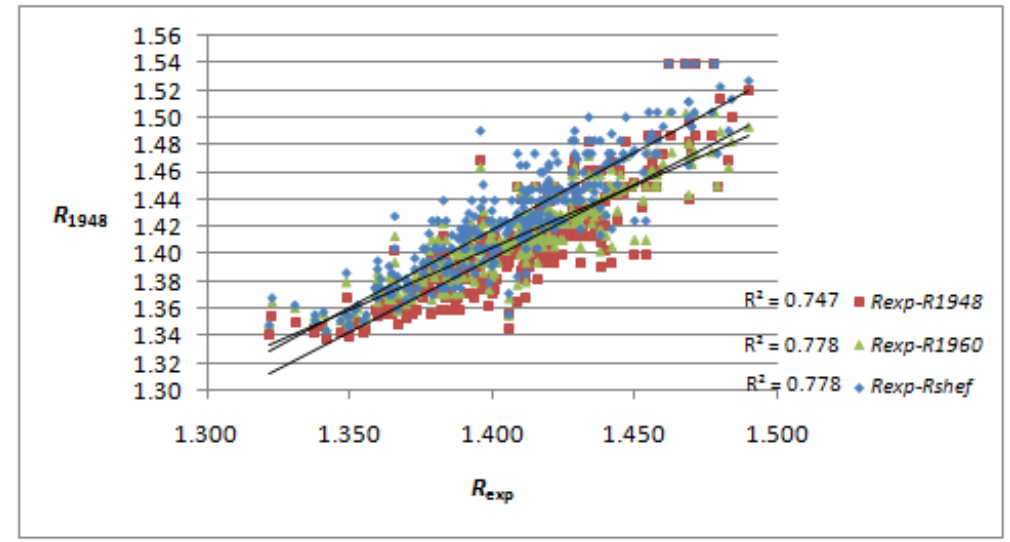

Figure5. The correlation between experimental and predicted bond lengths calculated using Pauling equations in 1948

c) The Calculations of Bond Order: The predicted Pauling bond orders PBOs were listed in table 1 . Then they correlated with the predicted bond lengths $R_{1948}, R_{1960}, R_{\text {new }}$ and $R_{\text {exp }}$, Fig. 6 . The figure revealed that for the set of benzenoids, the correlation between bond lengths and bond orders was decreasing and bent. This result confirmed the reverse correlation between bond orders and bond lengths.

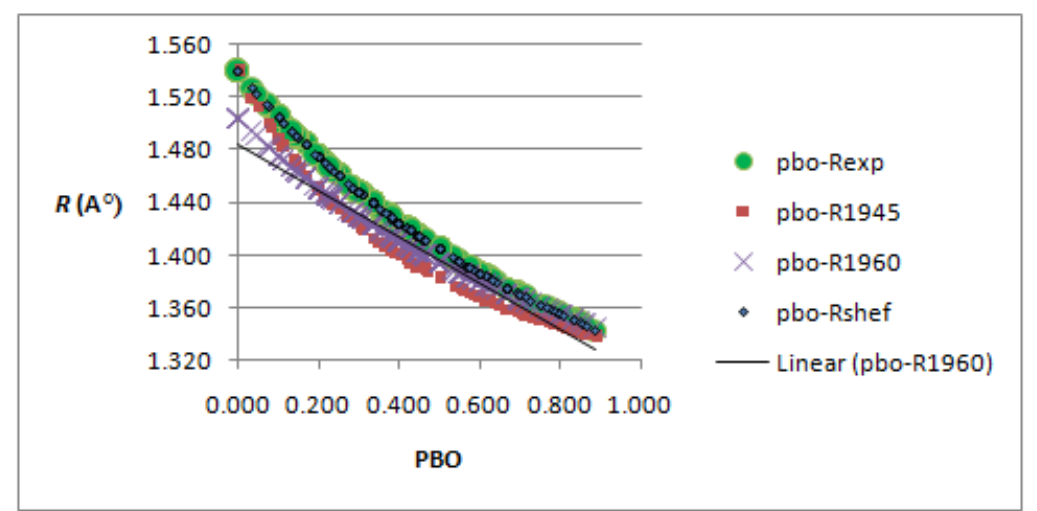

Figure6. The correlation between Pauling bond orders $P B O$ and corresponding bond lengths $R\left(R_{\text {exp }}, R_{1948,}\right.$ $R_{1960}$ and $R_{\text {new }}$ ) 
The Coulson bond orders of some non kekuléan conjugated hydrocarbons that calculated using SHMO program were drawn in Fig. 7. As shown, the values of them were zero and negative values.

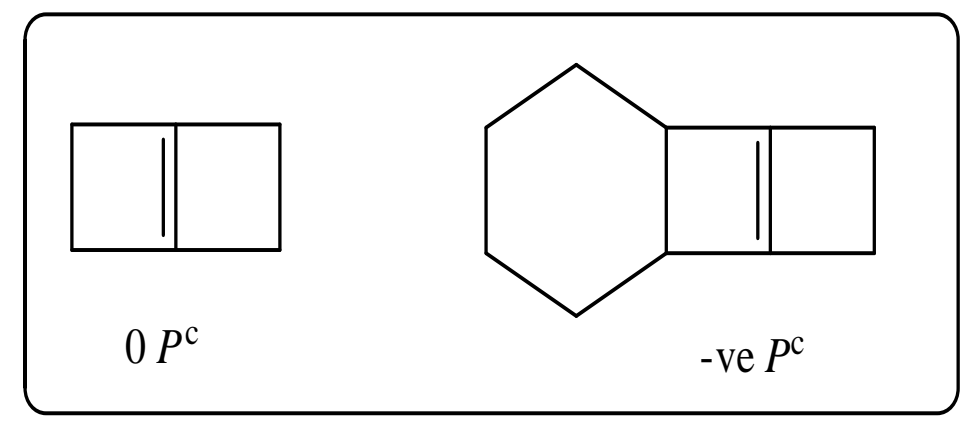

Figure7. The Coulson bond orders of two of non Kekuléan conjugated hydrocarbons

The Coulson bond orders CBOs of the set of benzenoids were calculated using the eq. (6). The results were listed in table (1). PBOs and CBOs were correlated with $R_{\text {exp }}$. The figures showed decreased linear correlations. The correlation of PBOs with $R_{\exp }$ has $R^{2}$ equal to 0.7812 whereas the correlation of CBOs with $R_{\exp }$ has $R^{2}$ equal to 0.7989 . It seems that the later correlation is closer to experiment. These figures also showed that the PBOs are zero for single bonds of bridged ring in perylene KF9, quaterlyene KF19 and dibenzoperylene KF24. But for the same bonds, the CBOs were higher than zero. This result could due to the nature of calculations of definition of Pauling and Coulson bond order because the former definition depends on the existence of Kekulè structure whereas the later definition depends on the sum of coefficients of the atoms of each bond.
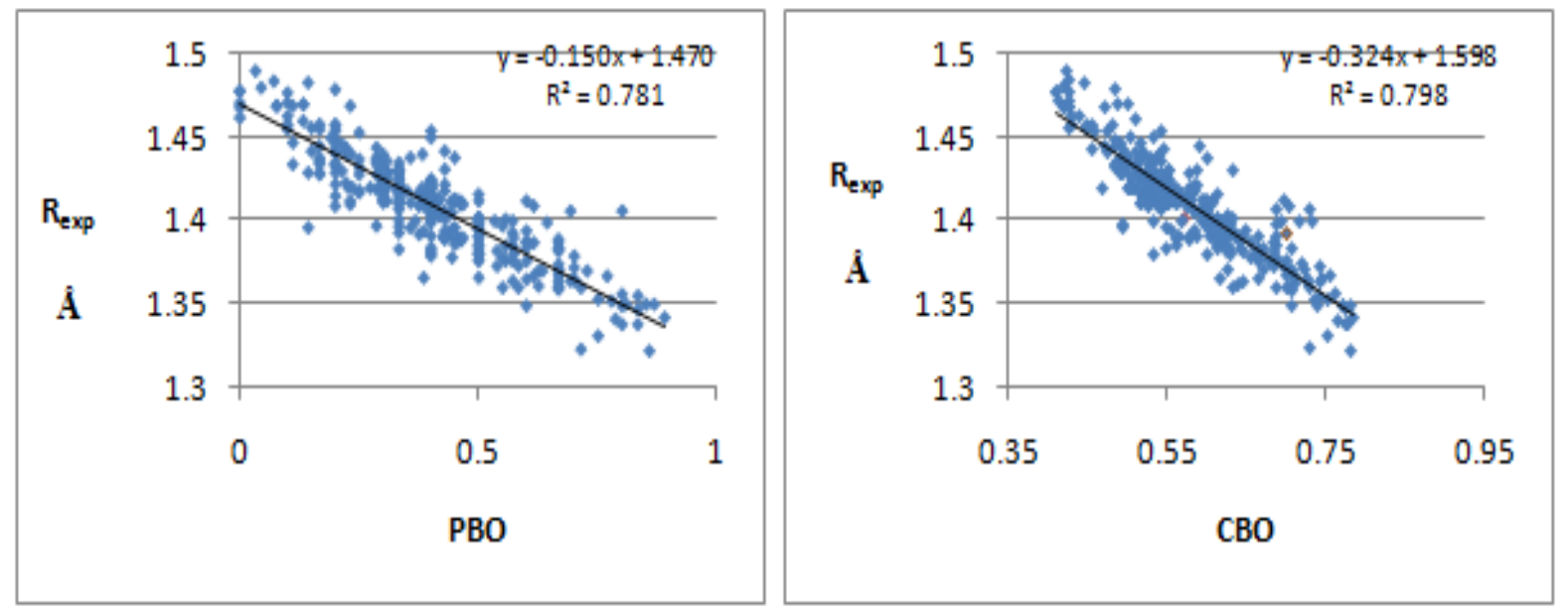

Figure8. (The left side) the correlation between Pauling bond order PBO and experimental bond lengths $R_{\text {exp }}$ and (the right side) the correlation between Coulson bond orders $C B O$ and experimental bond lengths $R_{\text {exp. }}$

As shown in Fig. 9, for each molecule, the individual correlations between Pauling bond order and experimental bond lengths (PBO- $R_{\text {exp }}$ ), Coulson bond order and experimental bond lengths (CBO$\left.R_{\text {exp }}\right)$, Pauling bond order and theoretical bond lengths (PBO- $\left.R_{\text {theo }}\right)$ and Coulson bond order and theoretical bond lengths(CBO- $\left.R_{\text {theo }}\right)$ are carried out to investigate the differentiation of both types of bond order, Pauling and Coulson. These correlations showed that, for the same molecule KF4there was two bonds with the same PBO and CBO but different values of $R_{\text {exp }}$. Unlike, for the same molecule KF5 there were two bonds with the same $R_{\exp }$ and different PBO but these bonds were with different CBO and different $R_{\text {exp. }}$. Kiralj et al. [13] assigned this degeneration in experimental data to the structural variations in the chemical bond, packing forces, unknown experimental errors and other effects. The correlation between PBO and $R_{\text {theo }}$ of naphthalene KF2 showed there are two types of bond but the correlation between $\mathrm{CBO}$ and $R_{\text {theo }}$ of the molecule showed four ypes of bonds. The same outcome can be noticed in table (1). These results indicate that CBO was more differentiated than PBO. This could be explained as previously according to the nature of Pauling and Coulson definition of bond order. The individual correlations between (CBO and $\left.R_{\exp }\right)$ and $\left(R_{\text {theo }}\right.$ and $\left.R_{\exp }\right)$ give the same number of bonds. This result indicates the similarity between Coulson and $a b$ initio definition of bond order. This similarity could due to the Coulson density matrix which is used in ab initio calculations of bond order. 
A Comparative Study of Bond Order and Bond Length Calculations of Some Conjugated Hydrocarbons using Two Different Methods

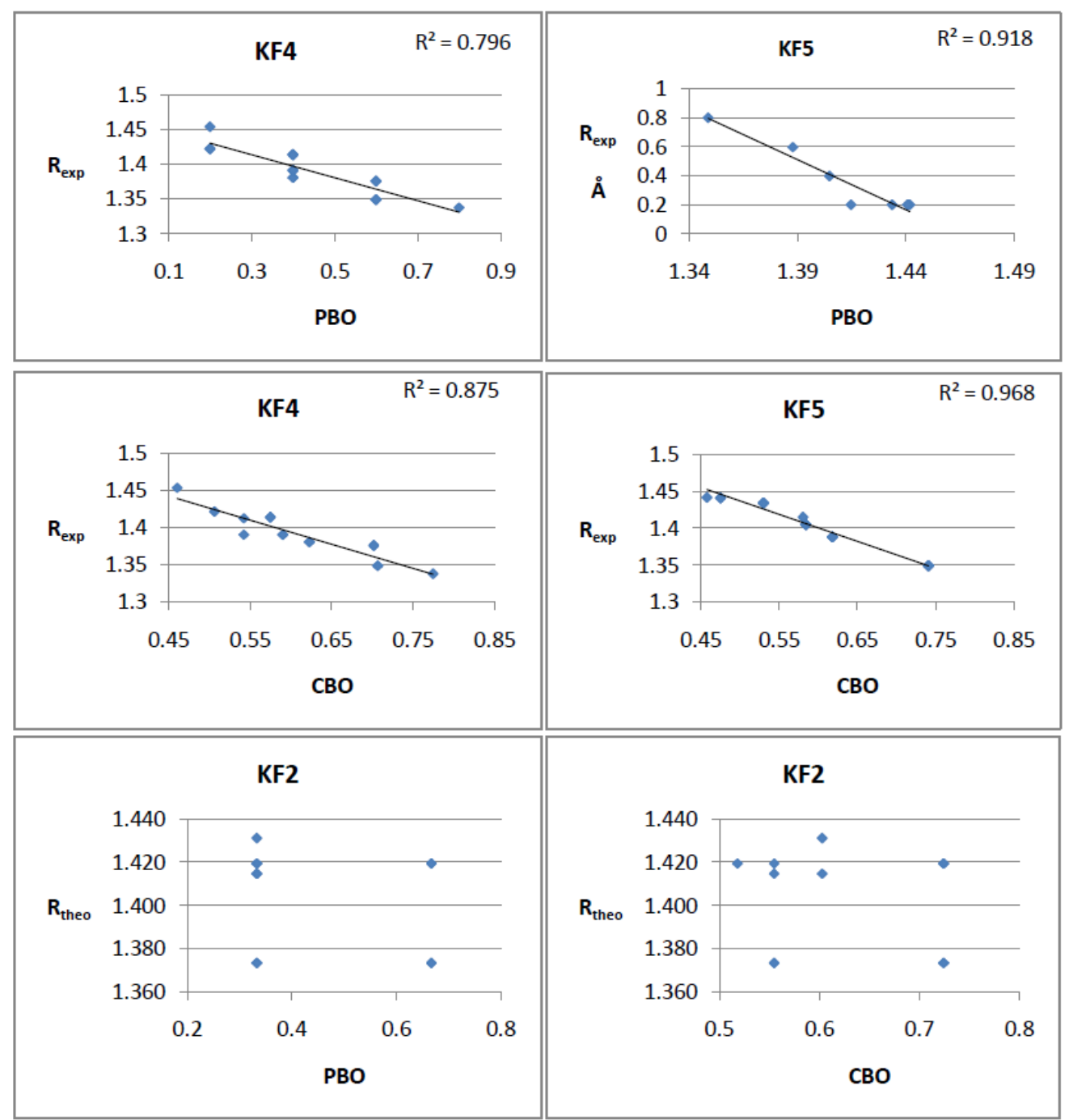

Figure9. The individual correlations between Pauling bond orders ( $1^{\text {st }}$ row) and experimental bond lengths, Coulson and experimental bond lengths $\left(2^{\text {nd }}\right.$ row) and Pauling and Coulson bond orders with theoretical bond lengths calculated by using Gaussian 09, DFT, B3LYP ( $3^{\text {rd }}$ row)

Table1. The Predicted Pauling bond orderspbo, their relative bond lengths $R_{n e w}, R_{1945,} R_{1960}$ and Coulson bond orderscbo, of the unique bonds of the set of molecules with their symbolic and symmetries.

\begin{tabular}{|c|c|c|c|c|c|c|c|c|c|c|}
\hline \multirow{2}{*}{ Seq } & \multirow{2}{*}{ Mol } & \multirow{2}{*}{$\begin{array}{l}\text { Name of } \\
\text { molecule }\end{array}$} & \multirow{2}{*}{ Sym } & \multirow{2}{*}{$\begin{array}{l}\text { Uniq- } \\
\text { uebond }\end{array}$} & \multirow{2}{*}{$R_{\text {exp }} / \mathbf{A}[15]$} & \multicolumn{5}{|c|}{ Present work } \\
\hline & & & & & & $\boldsymbol{R}_{\text {new }}$ & $R_{1945}$ & $R_{1960}$ & $\mathrm{CBO}$ & PBO \\
\hline 1 & KF1 & benzene & $D_{6 h}$ & A & 1.390 & 1.403 & 1.382 & 1.393 & 0.667 & 0.500 \\
\hline 2 & KF2 & napthalene & $D_{2 h}$ & $\mathrm{C}$ & 1.422 & 1.439 & 1.414 & 1.422 & 0.555 & 0.333 \\
\hline 3 & & & & $\mathrm{~B}$ & 1.371 & 1.375 & 1.359 & 1.370 & 0.725 & 0.667 \\
\hline 4 & & & & $\mathrm{~A}$ & 1.407 & 1.439 & 1.414 & 1.422 & 0.603 & 0.333 \\
\hline 5 & & & & $\mathrm{D}$ & 1.420 & 1.439 & 1.414 & 1.422 & 0.518 & 0.333 \\
\hline 6 & KF3 & anthracene & $D_{2 h}$ & $\mathrm{C}$ & 1.428 & 1.460 & 1.435 & 1.439 & 0.535 & 0.250 \\
\hline 7 & & & & $\mathrm{~A}$ & 1.395 & 1.404 & 1.382 & 1.393 & 0.606 & 0.500 \\
\hline 8 & & & & $\mathrm{D}$ & 1.353 & 1.362 & 1.351 & 1.360 & 0.737 & 0.750 \\
\hline 9 & & & & $\mathrm{E}$ & 1.418 & 1.460 & 1.435 & 1.439 & 0.586 & 0.250 \\
\hline 10 & & & & $\mathrm{~B}$ & 1.432 & 1.460 & 1.435 & 1.439 & 0.485 & 0.250 \\
\hline 11 & KF4 & phenanthrene & $\mathrm{C}_{2 \mathrm{v}}$ & G & 1.376 & 1.386 & 1.368 & 1.379 & 0.702 & 0.600 \\
\hline 12 & & & & $\mathrm{~F}$ & 1.381 & 1.424 & 1.400 & 1.410 & 0.623 & 0.400 \\
\hline 13 & & & & $\mathrm{E}$ & 1.349 & 1.395 & 1.368 & 1.379 & 0.707 & 0.600 \\
\hline 14 & & & & $\mathrm{D}$ & 1.414 & 1.424 & 1.400 & 1.410 & 0.575 & 0.400 \\
\hline 15 & & & & B & 1.422 & 1.474 & 1.450 & 1.450 & 0.506 & 0.200 \\
\hline 16 & & & & A & 1.338 & 1.355 & 1.346 & 1.354 & 0.775 & 0.800 \\
\hline 17 & & & & $\mathrm{C}$ & 1.413 & 1.424 & 1.400 & 1.400 & 0.542 & 0.400 \\
\hline
\end{tabular}


Manal O. Hamzah

\begin{tabular}{|c|c|c|c|c|c|c|c|c|c|c|}
\hline 18 & & & & $\mathrm{H}$ & 1.391 & 1.424 & 1.400 & 1.410 & 0.590 & 0.400 \\
\hline 19 & & & & I & 1.454 & 1.474 & 1.450 & 1.450 & 0.461 & 0.200 \\
\hline 20 & KF5 & tetracene & $\mathrm{D}_{2 \mathrm{~h}}$ & $\mathrm{E}$ & 1.434 & 1.474 & 1.450 & 1.450 & 0.530 & 0.200 \\
\hline 21 & & & & $\mathrm{C}$ & 1.388 & 1.386 & 1.368 & 1.379 & 0.618 & 0.600 \\
\hline 22 & & & & B & 1.405 & 1.424 & 1.400 & 1.410 & 0.584 & 0.400 \\
\hline 23 & & & & $\mathrm{~F}$ & 1.349 & 1.355 & 1.346 & 1.354 & 0.741 & 0.800 \\
\hline 24 & & & & $\mathrm{G}$ & 1.415 & 1.474 & 1.450 & 1.450 & 0.581 & 0.200 \\
\hline 25 & & & & $\mathrm{D}$ & 1.441 & 1.474 & 1.450 & 1.450 & 0.475 & 0.200 \\
\hline 26 & & & & A & 1.442 & 1.474 & 1.450 & 1.450 & 0.458 & 0.200 \\
\hline 27 & KF6 & triphynelene & $\mathrm{D}_{3 \mathrm{~h}}$ & $\mathrm{D}$ & 1.385 & 1.394 & 1.374 & 1.385 & 0.690 & 0.556 \\
\hline 28 & & & & $\mathrm{C}$ & 1.405 & 1.415 & 1.391 & 1.402 & 0.603 & 0.444 \\
\hline 29 & & & & A & 1.469 & 1.501 & 1.482 & 1.472 & 0.428 & 0.111 \\
\hline 30 & & & & $E$ & 1.390 & 1.415 & 1.391 & 1.402 & 0.637 & 0.444 \\
\hline 31 & & & & $\mathrm{~B}$ & 1.411 & 1.415 & 1.391 & 1.402 & 0.562 & 0.444 \\
\hline 32 & KF7 & chrysene & $\mathrm{C}_{2 \mathrm{~h}}$ & $\mathrm{~F}$ & 1.415 & 1.430 & 1.405 & 1.414 & 0.568 & 0.375 \\
\hline 33 & & & & $\mathrm{D}$ & 1.417 & 1.460 & 1.435 & 1.439 & 0.521 & 0.250 \\
\hline 34 & & & & $\mathrm{C}$ & 1.331 & 1.362 & 1.351 & 1.360 & 0.754 & 0.750 \\
\hline 35 & & & & B & 1.437 & 1.460 & 1.435 & 1.439 & 0.538 & 0.250 \\
\hline 36 & & & & $\mathrm{~K}$ & 1.453 & 1.460 & 1.435 & 1.439 & 0.476 & 0.250 \\
\hline 37 & & & & $\mathrm{~J}$ & 1.409 & 1.430 & 1.405 & 1.414 & 0.583 & 0.375 \\
\hline 38 & & & & I & 1.370 & 1.382 & 1.365 & 1.375 & 0.707 & 0.625 \\
\hline 39 & & & & $\mathrm{H}$ & 1.392 & 1.430 & 1.405 & 1.414 & 0.617 & 0.375 \\
\hline 40 & & & & $\mathrm{G}$ & 1.361 & 1.382 & 1.365 & 1.375 & 0.712 & 0.625 \\
\hline 41 & & & & $\mathrm{E}$ & 1.417 & 1.430 & 1.405 & 1.414 & 0.535 & 0.375 \\
\hline 42 & & & & $\mathrm{~A}$ & 1.402 & 1.404 & 1.382 & 1.393 & 0.573 & 0.500 \\
\hline 43 & KF8 & pyrene & $\mathrm{D}_{2 \mathrm{~h}}$ & $\mathrm{~A}$ & 1.347 & 1.351 & 1.341 & 1.350 & 0.777 & 0.833 \\
\hline 44 & & & & B & 1.429 & 1.484 & 1.461 & 1.458 & 0.503 & 0.167 \\
\hline 45 & & & & $\mathrm{D}$ & 1.400 & 1.404 & 1.382 & 1.393 & 0.594 & 0.500 \\
\hline 46 & & & & $E$ & 1.380 & 1.404 & 1.382 & 1.393 & 0.669 & 0.500 \\
\hline 47 & & & & $\mathrm{C}$ & 1.417 & 1.439 & 1.414 & 1.425 & 0.524 & 0.333 \\
\hline 48 & & & & $\mathrm{~F}$ & 1.423 & 1.439 & 1.414 & 1.422 & 0.536 & 0.333 \\
\hline 49 & KF9 & perylene & $\mathrm{D}_{2 \mathrm{~h}}$ & $\mathrm{D}$ & 1.393 & 1.439 & 1.414 & 1.422 & 0.629 & 0.333 \\
\hline 50 & & & & $\mathrm{G}$ & 1.471 & 1.540 & 1.540 & 1.504 & 0.414 & 0.000 \\
\hline 51 & & & & $E$ & 1.384 & 1.375 & 1.359 & 1.370 & 0.644 & 0.667 \\
\hline 52 & & & & $\mathrm{C}$ & 1.359 & 1.375 & 1.359 & 1.370 & 0.707 & 0.667 \\
\hline 53 & & & & B & 1.411 & 1.439 & 1.411 & 1.422 & 0.552 & 0.333 \\
\hline 54 & & & & $\mathrm{~F}$ & 1.429 & 1.439 & 1.414 & 1.422 & 0.529 & 0.333 \\
\hline 55 & & & & $\mathrm{~A}$ & 1.426 & 1.439 & 1.414 & 1.422 & 0.526 & 0.333 \\
\hline 56 & KF10 & $\begin{array}{c}1,2,5,6- \\
\text { dibenzoanthracene }\end{array}$ & $\mathrm{C}_{2 \mathrm{~h}}$ & I & 1.403 & 1.421 & 1.396 & 1.409 & 0.626 & 0.417 \\
\hline 57 & & & & $\mathrm{H}$ & 1.360 & 1.389 & 1.370 & 1.381 & 0.703 & 0.583 \\
\hline 58 & & & & $\mathrm{G}$ & 1.412 & 1.421 & 1.396 & 1.407 & 0.579 & 0.417 \\
\hline 59 & & & & $E$ & 1.436 & 1.484 & 1.461 & 1.458 & 0.499 & 0.167 \\
\hline 60 & & & & $\mathrm{D}$ & 1.338 & 1.351 & 1.343 & 1.350 & 0.778 & 0.833 \\
\hline 61 & & & & $\mathrm{C}$ & 1.438 & 1.484 & 1.461 & 1.458 & 0.501 & 0.167 \\
\hline 62 & & & & A & 1.391 & 1.404 & 1.382 & 1.393 & 0.605 & 0.500 \\
\hline 63 & & & & $M$ & 1.397 & 1.404 & 1.382 & 1.393 & 0.629 & 0.500 \\
\hline 64 & & & & $\mathrm{~L}$ & 1.455 & 1.484 & 1.461 & 1.458 & 0.451 & 0.167 \\
\hline 65 & & & & $\mathrm{~K}$ & 1.406 & 1.421 & 1.396 & 1.407 & 0.595 & 0.417 \\
\hline 66 & & & & $\mathrm{~J}$ & 1.373 & 1.389 & 1.370 & 1.381 & 0.697 & 0.583 \\
\hline 67 & & & & $\mathrm{~F}$ & 1.413 & 1.421 & 1.396 & 1.407 & 0.544 & 0.417 \\
\hline 68 & & & & B & 1.426 & 1.439 & 1.414 & 1.422 & 0.513 & 0.333 \\
\hline 69 & KF11 & 3,4-benzopyrene & $\mathrm{C}_{\mathrm{s}}$ & $\mathrm{N}$ & 1.364 & 1.375 & 1.359 & 1.370 & 0.710 & 0.667 \\
\hline 70 & & & & $\bar{M}$ & 1.397 & 1.439 & 1.414 & 1.422 & 0.609 & 0.333 \\
\hline 71 & & & & $\mathrm{~L}$ & 1.374 & 1.375 & 1.359 & 1.370 & 0.721 & 0.667 \\
\hline 72 & & & & $\mathrm{~K}$ & 1.425 & 1.439 & 1.414 & 1.422 & 0.552 & 0.333 \\
\hline 73 & & & & I & 1.419 & 1.439 & 1.414 & 1.422 & 0.562 & 0.333 \\
\hline 74 & & & & $\mathrm{H}$ & 1.361 & 1.375 & 1.359 & 1.370 & 0.641 & 0.667 \\
\hline 75 & & & & $\mathrm{~F}$ & 1.447 & 1.501 & 1.482 & 1.472 & 0.493 & 0.111 \\
\hline 76 & & & & $E$ & 1.342 & 1.343 & 1.338 & 1.344 & 0.784 & 0.889 \\
\hline
\end{tabular}


A Comparative Study of Bond Order and Bond Length Calculations of Some Conjugated Hydrocarbons using Two Different Methods

\begin{tabular}{|c|c|c|c|c|c|c|c|c|c|c|}
\hline 77 & & & & $\mathrm{D}$ & 1.434 & 1.501 & 1.482 & 1.472 & 0.493 & 0.111 \\
\hline 78 & & & & B & 1.401 & 1.394 & 1.374 & 1.385 & 0.604 & 0.556 \\
\hline 79 & & & & $\mathrm{~A}$ & 1.378 & 1.415 & 1.391 & 1.402 & 0.658 & 0.444 \\
\hline 80 & & & & $\mathrm{~W}$ & 1.376 & 1.394 & 1.374 & 1.385 & 0.680 & 0.556 \\
\hline 81 & & & & V & 1.412 & 1.415 & 1.391 & 1.402 & 0.580 & 0.444 \\
\hline 82 & & & & $\mathrm{~T}$ & 1.441 & 1.468 & 1.443 & 1.445 & 0.520 & 0.222 \\
\hline 83 & & & & $\mathrm{~S}$ & 1.352 & 1.358 & 1.348 & 1.356 & 0.754 & 0.778 \\
\hline 84 & & & & $\mathrm{R}$ & 1.423 & 1.468 & 1.443 & 1.445 & 0.539 & 0.222 \\
\hline 85 & & & & $\mathrm{O}$ & 1.419 & 1.439 & 1.414 & 1.422 & 0.580 & 0.333 \\
\hline 86 & & & & $\mathrm{~J}$ & 1.410 & 1.439 & 1.414 & 1.422 & 0.517 & 0.333 \\
\hline 87 & & & & $P$ & 1.435 & 1.439 & 1.414 & 1.422 & 0.489 & 0.333 \\
\hline 88 & & & & $\mathrm{G}$ & 1.444 & 1.468 & 1.443 & 1.445 & 0.486 & 0.222 \\
\hline 89 & & & & Q & 1.395 & 1.415 & 1.391 & 1.402 & 0.556 & 0.444 \\
\hline 90 & & & & $\bar{C}$ & 1.414 & 1.439 & 1.414 & 1.422 & 0.522 & 0.333 \\
\hline 91 & & & & $\mathrm{U}$ & 1.418 & 1.439 & 1.414 & 1.422 & 0.524 & 0.333 \\
\hline 92 & & & & $\mathrm{X}$ & 1.419 & 1.439 & 1.414 & 1.422 & 0.538 & 0.333 \\
\hline 93 & KF12 & picene & $\mathrm{C}_{2 \mathrm{v}}$ & $\mathrm{N}$ & 1.406 & 1.371 & 1.357 & 1.367 & 0.732 & 0.692 \\
\hline 94 & & & & $M$ & 1.430 & 1.446 & 1.420 & 1.427 & 0.554 & 0.308 \\
\hline 95 & & & & $\mathrm{~L}$ & 1.469 & 1.465 & 1.440 & 1.443 & 0.471 & 0.231 \\
\hline 96 & & & & $\mathrm{~K}$ & 1.394 & 1.428 & 1.403 & 1.413 & 0.586 & 0.385 \\
\hline 97 & & & & $\mathrm{~J}$ & 1.409 & 1.383 & 1.366 & 1.377 & 0.705 & 0.615 \\
\hline 98 & & & & I & 1.366 & 1.428 & 1.403 & 1.413 & 0.619 & 0.385 \\
\hline 99 & & & & $\mathrm{H}$ & 1.367 & 1.383 & 1.366 & 1.377 & 0.710 & 0.615 \\
\hline 100 & & & & $\mathrm{~F}$ & 1.414 & 1.428 & 1.403 & 1.413 & 0.537 & 0.385 \\
\hline 101 & & & & $\mathrm{G}$ & 1.414 & 1.428 & 1.403 & 1.413 & 0.570 & 0.385 \\
\hline 102 & & & & $E$ & 1.410 & 1.465 & 1.440 & 1.443 & 0.517 & 0.231 \\
\hline 103 & & & & D & 1.367 & 1.359 & 1.349 & 1.357 & 0.758 & 0.769 \\
\hline 104 & & & & B & $\begin{array}{l}1.388 \\
\end{array}$ & \begin{tabular}{|l|l|}
1.411 & \\
\end{tabular} & \begin{tabular}{|l|l|}
1.388 \\
\end{tabular} & \begin{tabular}{l|l}
1.399 \\
\end{tabular} & 0.563 & 0.462 \\
\hline 105 & & & & $\mathrm{C}$ & 1.412 & 1.465 & 1.440 & 1.443 & 0.532 & 0.231 \\
\hline 106 & & & & A & 1.429 & 1.446 & 1.420 & 1.427 & 0.493 & 0.308 \\
\hline 107 & KF13 & $\begin{array}{c}\text { dibenzo }[\mathrm{a}, \mathrm{c}] \\
\text { anthracene }\end{array}$ & $\mathrm{C}_{2 \mathrm{v}}$ & B & 1.389 & 1.411 & 1.388 & 1.399 & 0.640 & 0.462 \\
\hline 108 & & & & $\mathrm{C}$ & 1.373 & 1.397 & 1.376 & 1.388 & 0.686 & 0.538 \\
\hline 109 & & & & $\mathrm{D}$ & 1.412 & 1.411 & 1.388 & 1.399 & 0.607 & 0.462 \\
\hline 110 & & & & $\mathrm{~F}$ & 1.469 & 1.512 & 1.498 & 1.481 & 0.420 & 0.077 \\
\hline 111 & & & & $\mathrm{~A}$ & 1.382 & 1.397 & 1.376 & 1.388 & 0.686 & 0.538 \\
\hline 112 & & & & $\mathrm{E}$ & 1.410 & 1.411 & 1.388 & 1.399 & 0.564 & 0.462 \\
\hline 113 & & & & $\mathrm{G}$ & 1.410 & 1.411 & 1.388 & 1.399 & 0.607 & 0.462 \\
\hline 114 & & & & $\mathrm{H}$ & 1.469 & 1.512 & 1.498 & 1.481 & 0.420 & 0.077 \\
\hline 115 & & & & $\mathrm{~J}$ & 1.387 & 1.383 & 1.366 & 1.377 & 0.658 & 0.615 \\
\hline 116 & & & & $\mathrm{~K}$ & 1.409 & 1.428 & 1.403 & 1.413 & 0.575 & 0.385 \\
\hline 117 & & & & $\mathrm{M}$ & 1.426 & 1.446 & 1.420 & 1.427 & 0.549 & 0.308 \\
\hline 118 & & & & $\mathrm{~N}$ & 1.364 & 1.371 & 1.357 & 1.367 & 0.727 & 0.692 \\
\hline 119 & & & & $\mathrm{O}$ & 1.412 & 1.446 & 1.420 & 1.427 & 0.598 & 0.308 \\
\hline 120 & & & & $\mathrm{~L}$ & 1.420 & 1.446 & 1.420 & 1.427 & 0.502 & 0.308 \\
\hline 121 & & & & I & 1.436 & 1.446 & 1.420 & 1.427 & 0.513 & 0.308 \\
\hline 122 & KF14 & $\begin{array}{l}\text { dibenzo [fg,op] } \\
\text { tetracene }\end{array}$ & $\mathrm{D}_{2 \mathrm{~h}}$ & I & 1.383 & 1.404 & 1.382 & 1.393 & 0.667 & 0.500 \\
\hline 123 & & & & $\mathrm{H}$ & 1.416 & 1.404 & 1.382 & 1.393 & 0.614 & 0.500 \\
\hline 124 & & & & $E$ & 1.477 & 1.504 & 1.487 & 1.475 & 0.428 & 0.100 \\
\hline 125 & & & & $\mathrm{C}$ & 1.438 & 1.414 & 1.390 & 1.401 & 0.603 & 0.450 \\
\hline 126 & & & & B & 1.397 & 1.395 & 1.375 & 1.386 & 0.690 & 0.550 \\
\hline 127 & & & & $\mathrm{~A}$ & 1.391 & 1.414 & 1.390 & 1.401 & 0.637 & 0.450 \\
\hline 128 & & & & D & 1.386 & 1.414 & 1.390 & 1.401 & 0.562 & 0.450 \\
\hline 129 & & & & $\mathrm{~F}$ & 1.416 & 1.424 & 1.400 & 1.410 & 0.549 & 0.400 \\
\hline 130 & & & & $\mathrm{G}$ & 1.457 & 1.474 & 1.450 & 1.450 & 0.482 & 0.200 \\
\hline 131 & KF15 & pentacene & $\mathrm{D}_{2 \mathrm{~h}}$ & $\mathrm{C}$ & 1.434 & 1.484 & 1.461 & 1.458 & 0.529 & 0.167 \\
\hline 132 & & & & $\mathrm{E}$ & 1.387 & 1.375 & 1.395 & 1.370 & 0.622 & 0.667 \\
\hline 133 & & & & $\mathrm{~F}$ & 1.412 & 1.439 & 1.414 & 1.422 & 0.578 & 0.333 \\
\hline
\end{tabular}


Manal O. Hamzah

\begin{tabular}{|c|c|c|c|c|c|c|c|c|c|c|}
\hline 134 & & & & $\mathrm{H}$ & 1.412 & 1.404 & 1.382 & 1.393 & 0.596 & 0.500 \\
\hline 135 & & & & B & 1.355 & 1.351 & 1.343 & 1.350 & 0.742 & 0.833 \\
\hline 136 & & & & $\mathrm{~A}$ & 1.428 & 1.484 & 1.461 & 1.458 & 0.579 & 0.167 \\
\hline 137 & & & & $\mathrm{D}$ & 1.445 & 1.484 & 1.461 & 1.458 & 0.472 & 0.167 \\
\hline 138 & & & & G & 1.458 & 1.484 & 1.461 & 1.458 & 0.451 & 0.167 \\
\hline 139 & KF16 & $\begin{array}{l}\text { benzo [ghi] } \\
\text { perylene }\end{array}$ & $\mathrm{C}_{2 \mathrm{v}}$ & $\mathrm{L}$ & 1.394 & 1.418 & 1.394 & 1.405 & 0.649 & 0.429 \\
\hline 140 & & & & $\mathrm{~K}$ & 1.376 & 1.391 & 1.371 & 1.382 & 0.688 & 0.571 \\
\hline 141 & & & & $\mathrm{~J}$ & 1.389 & 1.418 & 1.394 & 1.405 & 0.579 & 0.429 \\
\hline 142 & & & & G & 1.430 & 1.470 & 1.445 & 1.447 & 0.513 & 0.214 \\
\hline 143 & & & & $\mathrm{~F}$ & 1.341 & 1.357 & 1.374 & 1.355 & 0.765 & 0.786 \\
\hline 144 & & & & $\mathrm{E}$ & 1.446 & 1.470 & 1.445 & 1.447 & 0.519 & 0.214 \\
\hline 145 & & & & B & 1.396 & 1.434 & 1.408 & 1.418 & 0.550 & 0.357 \\
\hline 146 & & & & $\mathrm{~A}$ & 1.399 & 1.379 & 1.362 & 1.373 & 0.734 & 0.643 \\
\hline 147 & & & & $\mathrm{M}$ & 1.385 & 1.391 & 1.371 & 1.383 & 0.627 & 0.571 \\
\hline 148 & & & & $\mathrm{O}$ & 1.484 & 1.514 & 1.500 & 1.482 & 0.427 & 0.071 \\
\hline 149 & & & & $\mathrm{H}$ & 1.438 & 1.434 & 1.408 & 1.418 & 0.530 & 0.357 \\
\hline 150 & & & & $\mathrm{~N}$ & 1.411 & 1.434 & 1.408 & 1.418 & 0.537 & 0.357 \\
\hline 151 & & & & $\mathrm{C}$ & 1.406 & 1.418 & 1.394 & 1.405 & 0.545 & 0.429 \\
\hline 152 & & & & I & 1.419 & 1.451 & 1.425 & 1.431 & 0.516 & 0.286 \\
\hline 153 & & & & $\mathrm{D}$ & 1.438 & 1.451 & 1.425 & 1.431 & 0.519 & 0.286 \\
\hline 154 & KF17 & coronene & $D_{6 h}$ & $\mathrm{C}$ & 1.414 & 1.447 & 1.421 & 1.429 & 0.538 & 0.300 \\
\hline 155 & & & & $\mathrm{D}$ & 1.372 & 1.370 & 1.356 & 1.366 & 0.745 & 0.700 \\
\hline 156 & & & & $\mathrm{~B}$ & 1.420 & 1.424 & 1.400 & 1.410 & 0.538 & 0.400 \\
\hline 157 & & & & A & 1.424 & 1.447 & 1.421 & 1.429 & 0.522 & 0.300 \\
\hline 158 & KF18 & $\begin{array}{c}\text { benzo [fg,gi] } \\
\text { phenanthro } \\
{[9,10,1,2,3 \text {-pqrst] }} \\
\text { pentaphene }\end{array}$ & $\mathrm{C}_{2 \mathrm{v}}$ & Q & 1.378 & 1.395 & 1.375 & 1.386 & 0.689 & 0.550 \\
\hline 159 & & & & $\mathrm{R}$ & 1.396 & 1.414 & 1.390 & 1.401 & 0.603 & 0.450 \\
\hline 160 & & & & $\mathrm{~S}$ & 1.471 & 1.504 & 1.487 & 1.475 & 0.428 & 0.100 \\
\hline 161 & & & & $\mathrm{~T}$ & 1.391 & 1.404 & 1.382 & 1.393 & 0.621 & 0.500 \\
\hline 162 & & & & $\mathrm{P}$ & 1.392 & 1.414 & 1.390 & 1.401 & 0.637 & 0.450 \\
\hline 163 & & & & $\mathrm{O}$ & 1.360 & 1.395 & 1.375 & 1.386 & 0.689 & 0.550 \\
\hline 164 & & & & $\mathrm{M}$ & 1.413 & 1.414 & 1.390 & 1.401 & 0.561 & 0.450 \\
\hline 165 & & & & $\mathrm{~N}$ & 1.413 & 1.414 & 1.390 & 1.401 & 0.603 & 0.450 \\
\hline 166 & & & & $\mathrm{~L}$ & 1.455 & 1.504 & 1.487 & 1.475 & 0.428 & 0.100 \\
\hline 167 & & & & $\mathrm{~J}$ & 1.401 & 1.404 & 1.382 & 1.393 & 0.615 & 0.500 \\
\hline 168 & & & & I & 1.366 & 1.404 & 1.382 & 1.393 & 0.666 & 0.500 \\
\hline 169 & & & & $\mathrm{H}$ & 1.377 & 1.404 & 1.382 & 1.393 & 0.667 & 0.500 \\
\hline 170 & & & & G & 1.402 & 1.404 & 1.382 & 1.393 & 0.613 & 0.500 \\
\hline 171 & & & & $\mathrm{E}$ & 1.463 & 1.504 & 1.487 & 1.475 & 0.430 & 0.100 \\
\hline 172 & & & & B & 1.386 & 1.404 & 1.382 & 1.393 & 0.613 & 0.500 \\
\hline 173 & & & & A & 1.381 & 1.404 & 1.382 & 1.393 & 0.667 & 0.500 \\
\hline 174 & & & & $\mathrm{U}$ & 1.408 & 1.424 & 1.400 & 1.410 & 0.540 & 0.400 \\
\hline 175 & & & & $\mathrm{~K}$ & 1.425 & 1.424 & 1.400 & 1.410 & 0.547 & 0.400 \\
\hline 176 & & & & $\mathrm{~F}$ & 1.411 & 1.424 & 1.400 & 1.410 & 0.548 & 0.400 \\
\hline 177 & & & & $\mathrm{~V}$ & 1.449 & 1.474 & 1.450 & 1.450 & 0.487 & 0.200 \\
\hline 178 & & & & $\mathrm{C}$ & 1.424 & 1.424 & 1.400 & 1.410 & 0.548 & 0.400 \\
\hline 179 & & & & $\mathrm{~W}$ & 1.424 & 1.424 & 1.400 & 1.410 & 0.546 & 0.400 \\
\hline 180 & & & & $\mathrm{D}$ & 1.433 & 1.474 & 1.450 & 1.450 & 0.486 & 0.200 \\
\hline 181 & KF19 & quatrylene & $D_{2 h}$ & $\mathrm{D}$ & 1.401 & 1.439 & 1.414 & 1.422 & 0.634 & 0.333 \\
\hline 182 & & & & $\mathrm{C}$ & 1.367 & 1.375 & 1.359 & 1.370 & 0.702 & 0.667 \\
\hline 183 & & & & $\mathrm{~B}$ & 1.417 & 1.439 & 1.414 & 1.422 & 0.552 & 0.333 \\
\hline 184 & & & & $\mathrm{G}$ & 1.468 & 1.540 & 1.540 & 1.504 & 0.429 & 0.000 \\
\hline 185 & & & & $\mathrm{E}$ & 1.382 & 1.375 & 1.359 & 1.370 & 0.632 & 0.667 \\
\hline 186 & & & & I & 1.383 & 1.375 & 1.359 & 1.370 & 0.616 & 0.667 \\
\hline 187 & & & & $\mathrm{~J}$ & 1.383 & 1.439 & 1.414 & 1.422 & 0.671 & 0.333 \\
\hline 188 & & & & $\mathrm{~L}$ & 1.462 & 1.540 & 1.540 & 1.504 & 0.441 & 0.000 \\
\hline 189 & & & & $\mathrm{~K}$ & 1.389 & 1.375 & 1.359 & 1.370 & 0.612 & 0.667 \\
\hline
\end{tabular}


A Comparative Study of Bond Order and Bond Length Calculations of Some Conjugated Hydrocarbons using Two Different Methods

\begin{tabular}{|c|c|c|c|c|c|c|c|c|c|c|}
\hline 190 & & & & $\mathrm{~A}$ & 1.420 & 1.439 & 1.414 & 1.422 & 0.526 & 0.333 \\
\hline 191 & & & & $\mathrm{~F}$ & 1.431 & 1.439 & 1.414 & 1.422 & 0.529 & 0.333 \\
\hline 192 & & & & $\mathrm{H}$ & 1.431 & 1.439 & 1.414 & 1.422 & 0.526 & 0.333 \\
\hline 193 & & & & $\mathrm{M}$ & 1.429 & 1.439 & 1.414 & 1.422 & 0.526 & 0.333 \\
\hline 194 & & & & $\mathrm{~N}$ & 1.431 & 1.439 & 1.414 & 1.422 & 0.535 & 0.333 \\
\hline 195 & KF20 & $\begin{array}{c}\text { hexabenzo } \\
\text { [bc,ef,hi,kl,no,gr] } \\
\text { coronene }\end{array}$ & $\mathrm{D}_{6 \mathrm{~h}}$ & $\mathrm{~F}$ & 1.376 & 1.404 & 1.382 & 1.393 & 0.667 & 0.500 \\
\hline 196 & & & & $\mathrm{E}$ & 1.398 & 1.404 & 1.382 & 1.393 & 0.613 & 0.500 \\
\hline 197 & & & & $\mathrm{D}$ & 1.458 & 1.504 & 1.487 & 1.475 & 0.431 & 0.100 \\
\hline 198 & & & & $\mathrm{C}$ & 1.417 & 1.424 & 1.400 & 1.410 & 0.547 & 0.400 \\
\hline 199 & & & & $\mathrm{~B}$ & 1.446 & 1.474 & 1.450 & 1.450 & 0.488 & 0.200 \\
\hline 200 & & & & A & 1.417 & 1.424 & 1.450 & 1.450 & 0.542 & 0.400 \\
\hline 201 & KF21 & kekulene & $D_{6 h}$ & B & 1.442 & 1.489 & 1.467 & 1.462 & 0.494 & 0.150 \\
\hline 202 & & & & $\mathrm{E}$ & 1.395 & 1.404 & 1.382 & 1.393 & 0.612 & 0.500 \\
\hline 203 & & & & $\mathrm{~A}$ & 1.350 & 1.348 & 1.341 & 1.348 & 0.781 & 0.850 \\
\hline 204 & & & & $\mathrm{C}$ & 1.418 & 1.435 & 1.410 & 1.419 & 0.514 & 0.350 \\
\hline 205 & & & & $\mathrm{D}$ & 1.456 & 1.489 & 1.467 & 1.462 & 0.450 & 0.150 \\
\hline 206 & & & & $\mathrm{~F}$ & 1.386 & 1.404 & 1.382 & 1.393 & 0.623 & 0.500 \\
\hline 207 & KF22 & $\begin{array}{c}\text { benzo }[1,2,3- \\
\text { bc: } 4,5,6-b, c] \\
\text { dicoronene }\end{array}$ & $\mathrm{D}_{2 \mathrm{~h}}$ & $\mathrm{~F}$ & 1.365 & 1.370 & 1.356 & 1.366 & 0.748 & 0.700 \\
\hline 208 & & & & $\mathrm{E}$ & 1.419 & 1.447 & 1.421 & 1.429 & 0.535 & 0.300 \\
\hline 209 & & & & $\mathrm{C}$ & 1.422 & 1.447 & 1.421 & 1.429 & 0.540 & 0.300 \\
\hline 210 & & & & $\mathrm{~B}$ & 1.364 & 1.370 & 1.356 & 1.366 & 0.743 & 0.700 \\
\hline 211 & & & & A & 1.417 & 1.447 & 1.421 & 1.429 & 0.538 & 0.300 \\
\hline 212 & & & & $\mathrm{G}$ & 1.424 & 1.447 & 1.421 & 1.429 & 0.530 & 0.300 \\
\hline 213 & & & & I & 1.413 & 1.447 & 1.421 & 1.429 & 0.561 & 0.300 \\
\hline 214 & & & & $\mathrm{~L}$ & 1.478 & 1.540 & 1.540 & 1.504 & 0.411 & 0.000 \\
\hline 215 & & & & $\mathrm{~J}$ & 1.379 & 1.370 & 1.356 & 1.366 & 0.664 & 0.700 \\
\hline 216 & & & & $\mathrm{D}$ & 1.415 & 1.424 & 1.400 & 1.410 & 0.535 & 0.400 \\
\hline 217 & & & & $\mathrm{Q}$ & 1.422 & 1.424 & 1.421 & 1.429 & 0.538 & 0.400 \\
\hline 218 & & & & $\mathrm{P}$ & 1.422 & 1.447 & 1.421 & 1.429 & 0.521 & 0.300 \\
\hline 219 & & & & $\mathrm{H}$ & 1.412 & 1.424 & 1.400 & 1.410 & 0.529 & 0.400 \\
\hline 220 & & & & $\mathrm{O}$ & 1.424 & 1.447 & 1.421 & 1.429 & 0.526 & 0.300 \\
\hline 221 & & & & $\mathrm{~K}$ & 1.432 & 1.447 & 1.421 & 1.429 & 0.512 & 0.300 \\
\hline 222 & & & & $\mathrm{~N}$ & 1.421 & 1.447 & 1.421 & 1.429 & 0.519 & 0.300 \\
\hline 223 & & & & $\mathrm{M}$ & 1.420 & 1.424 & 1.400 & 1.410 & 0.547 & 0.400 \\
\hline 224 & KF23 & benz[a]anthracene & $\mathrm{C}_{\mathrm{s}}$ & $\mathrm{O}$ & 1.436 & 1.451 & 1.425 & 1.431 & 0.542 & 0.286 \\
\hline 225 & & & & $\mathrm{M}$ & 1.431 & 1.418 & 1.394 & 1.405 & 0.590 & 0.429 \\
\hline 226 & & & & $\mathrm{~L}$ & 1.384 & 1.391 & 1.371 & 1.383 & 0.628 & 0.571 \\
\hline 227 & & & & $\mathrm{~J}$ & 1.429 & 1.491 & 1.469 & 1.464 & 0.494 & 0.143 \\
\hline 228 & & & & I & 1.322 & 1.347 & 1.341 & 1.348 & 0.783 & 0.857 \\
\hline 229 & & & & $\mathrm{H}$ & 1.396 & 1.491 & 1.469 & 1.464 & 0.494 & 0.143 \\
\hline 230 & & & & $\mathrm{~F}$ & 1.418 & 1.418 & 1.469 & 1.464 & 0.581 & 0.429 \\
\hline 231 & & & & $\mathrm{E}$ & 1.393 & 1.391 & 1.371 & 1.382 & 0.700 & 0.571 \\
\hline 232 & & & & $\mathrm{D}$ & 1.392 & 1.418 & 1.394 & 1.405 & 0.628 & 0.429 \\
\hline 233 & & & & $\mathrm{C}$ & 1.400 & 1.391 & 1.371 & 1.382 & 0.695 & 0.571 \\
\hline 234 & & & & $\mathrm{G}$ & 1.442 & 1.418 & & & 0.546 & 0.429 \\
\hline 235 & & & & B & 1.401 & 1.418 & 1.394 & 1.405 & 0.597 & 0.429 \\
\hline 236 & & & & $\mathrm{~K}$ & 1.434 & 1.451 & 1.425 & 1.431 & 0.500 & 0.286 \\
\hline 237 & & & & A & 1.483 & 1.491 & 1.469 & 1.464 & 0.447 & 0.143 \\
\hline 238 & & & & $\mathrm{U}$ & 1.364 & 1.391 & 1.371 & 1.383 & 0.646 & 0.571 \\
\hline 239 & & & & $\mathrm{~N}$ & 1.397 & 1.451 & 1.425 & 1.431 & 0.495 & 0.286 \\
\hline 240 & & & & $\mathrm{~T}$ & 1.422 & 1.418 & 1.394 & 1.405 & 0.584 & 0.429 \\
\hline 241 & & & & $\mathrm{~S}$ & 1.428 & 1.451 & 1.425 & 1.431 & 0.545 & 0.286 \\
\hline 242 & & & & $\mathrm{R}$ & 1.360 & 1.368 & 1.354 & 1.364 & 0.731 & 0.714 \\
\hline 243 & & & & $\mathrm{P}$ & 1.323 & 1.368 & 1.425 & 1.431 & 0.732 & 0.714 \\
\hline 244 & & & & $\mathrm{Q}$ & 1.444 & 1.451 & 1.425 & 1.431 & 0.593 & 0.286 \\
\hline
\end{tabular}


Manal O. Hamzah

\begin{tabular}{|c|c|c|c|c|c|c|c|c|c|c|}
\hline 245 & KF24 & $\begin{array}{c}2,3,8,9- \\
\text { dibenzoperylene }\end{array}$ & $\mathrm{C}_{2 \mathrm{~h}}$ & $\mathrm{D}$ & 1.387 & 1.386 & 1.368 & 1.379 & 0.686 & 0.600 \\
\hline 246 & & & & $\mathrm{C}$ & 1.422 & 1.424 & 1.400 & 1.410 & 0.589 & 0.400 \\
\hline 247 & & & & A & 1.458 & 1.474 & 1.450 & 1.450 & 0.456 & 0.200 \\
\hline 248 & & & & Q & 1.403 & 1.424 & 1.400 & 1.410 & 0.594 & 0.400 \\
\hline 249 & & & & $\mathrm{P}$ & 1.412 & 1.386 & 1.368 & 1.379 & 0.698 & 0.600 \\
\hline 250 & & & & $\mathrm{O}$ & 1.399 & 1.424 & 1.400 & 1.410 & 0.621 & 0.400 \\
\hline 251 & & & & $\mathrm{~N}$ & 1.384 & 1.386 & 1.368 & 1.379 & 0.709 & 0.600 \\
\hline 252 & & & & $\mathrm{~L}$ & 1.379 & 1.424 & 1.400 & 1.410 & 0.533 & 0.400 \\
\hline 253 & & & & M & 1.413 & 1.424 & 1.400 & 1.410 & 0.567 & 0.400 \\
\hline 254 & & & & $\mathrm{~K}$ & 1.409 & 1.474 & 1.450 & 1.450 & 0.529 & 0.200 \\
\hline 255 & & & & G & 1.478 & 1.540 & 1.540 & 1.504 & 0.411 & 0.000 \\
\hline 256 & & & & $\mathrm{~J}$ & 1.406 & 1.355 & 1.346 & 1.354 & 0.689 & 0.800 \\
\hline 257 & & & & $\mathrm{~F}$ & 1.394 & 1.386 & 1.368 & 1.379 & 0.632 & 0.600 \\
\hline 258 & & & & $\mathrm{E}$ & 1.381 & 1.424 & 1.400 & 1.410 & 0.647 & 0.400 \\
\hline 259 & & & & B & 1.384 & 1.424 & 1.400 & 1.410 & 0.550 & 0.400 \\
\hline 260 & & & & I & 1.479 & 1.474 & 1.450 & 1.450 & 0.486 & 0.200 \\
\hline 261 & & & & $\mathrm{H}$ & 1.454 & 1.424 & 1.400 & 1.410 & 0.544 & 0.400 \\
\hline 262 & KF25 & $\begin{array}{c}1,14- \\
\text { benzobisanthrene }\end{array}$ & $\mathrm{C}_{2 \mathrm{v}}$ & $\mathrm{F}$ & 1.470 & 1.494 & 1.473 & 1.466 & 0.501 & 0.133 \\
\hline 263 & & & & $\mathrm{H}$ & 1.370 & 1.380 & 1.363 & 1.374 & 0.626 & 0.633 \\
\hline 264 & & & & I & 1.400 & 1.432 & 1.406 & 1.416 & 0.570 & 0.367 \\
\hline 265 & & & & $\mathrm{~K}$ & 1.430 & 1.439 & 1.414 & 1.422 & 0.552 & 0.333 \\
\hline 266 & & & & $\mathrm{~L}$ & 1.370 & 1.375 & 1.359 & 1.370 & 0.703 & 0.667 \\
\hline 267 & & & & $\mathrm{M}$ & 1.430 & 1.439 & 1.414 & 1.422 & 0.633 & 0.333 \\
\hline 268 & & & & $\mathrm{~N}$ & 1.360 & 1.375 & 1.359 & 1.370 & 0.634 & 0.667 \\
\hline 269 & & & & $\mathrm{P}$ & 1.490 & 1.527 & 1.520 & 1.493 & 0.424 & 0.033 \\
\hline 270 & & & & $\mathrm{E}$ & 1.350 & 1.346 & 1.340 & 1.347 & 0.772 & 0.867 \\
\hline 271 & & & & $\mathrm{D}$ & 1.460 & 1.494 & 1.473 & 1.466 & 0.510 & 0.133 \\
\hline 272 & & & & B & 1.390 & 1.410 & 1.387 & 1.399 & 0.565 & 0.467 \\
\hline 273 & & & & A & 1.400 & 1.398 & 1.377 & 1.388 & 0.717 & 0.533 \\
\hline 274 & & & & G & 1.440 & 1.465 & 1.439 & 1.442 & 0.496 & 0.233 \\
\hline 275 & & & & $\mathrm{~J}$ & 1.420 & 1.447 & 1.421 & 1.429 & 0.509 & 0.300 \\
\hline 276 & & & & $\mathrm{O}$ & 1.430 & 1.447 & 1.421 & 1.429 & 0.532 & 0.300 \\
\hline 277 & & & & $\mathrm{C}$ & 1.420 & 1.424 & 1.400 & 1.410 & 0.534 & 0.400 \\
\hline 278 & & & & $\mathrm{~T}$ & 1.430 & 1.447 & 1.421 & 1.429 & 0.527 & 0.300 \\
\hline 279 & & & & $\mathrm{U}$ & 1.440 & 1.447 & 1.421 & 1.429 & 0.522 & 0.300 \\
\hline 280 & & & & $S$ & 1.410 & 1.410 & 1.387 & 1.399 & 0.551 & 0.467 \\
\hline 281 & & & & $\mathrm{Q}$ & 1.400 & 1.424 & 1.400 & 1.410 & 0.535 & 0.400 \\
\hline 282 & & & & R & 1.470 & 1.494 & 1.473 & 1.466 & 0.489 & 0.133 \\
\hline 283 & KF26 & ovalene & $\mathrm{C}_{2 \mathrm{v}}$ & $E$ & 1.441 & 1.474 & 1.450 & 1.450 & 0.511 & 0.200 \\
\hline 284 & & & & D & 1.400 & 1.404 & 1.382 & 1.393 & 0.604 & 0.500 \\
\hline 285 & & & & $F$ & 1.356 & 1.355 & 1.346 & 1.354 & 0.764 & 0.800 \\
\hline 286 & & & & $\mathrm{G}$ & 1.429 & 1.474 & 1.450 & 1.450 & 0.519 & 0.200 \\
\hline 287 & & & & I & 1.413 & 1.424 & 1.400 & 1.410 & 0.556 & 0.400 \\
\hline 288 & & & & $\mathrm{~J}$ & 1.365 & 1.386 & 1.368 & 1.379 & 0.726 & 0.600 \\
\hline 289 & & & & $\mathrm{C}$ & 1.424 & 1.447 & 1.421 & 1.429 & 0.508 & 0.300 \\
\hline 290 & & & & $\mathrm{H}$ & 1.450 & 1.424 & 1.400 & 1.410 & 0.535 & 0.400 \\
\hline 291 & & & & $\mathrm{~L}$ & 1.411 & 1.447 & 1.421 & 1.429 & 0.526 & 0.300 \\
\hline 292 & & & & $\mathrm{~K}$ & 1.413 & 1.447 & 1.421 & 1.429 & 0.521 & 0.300 \\
\hline 293 & & & & B & 1.415 & 1.424 & 1.400 & 1.410 & 0.541 & 0.400 \\
\hline 294 & & & & $\mathrm{~A}$ & 1.435 & 1.474 & 1.450 & 1.450 & 0.497 & 0.200 \\
\hline 295 & KF27 & $\begin{array}{l}\text { Tetrabenzo } \\
\text { [de,no,st,c,d] } \\
\text { heptacene }\end{array}$ & $\mathrm{D}_{2 \mathrm{~h}}$ & $\mathrm{~F}$ & 1.390 & 1.419 & 1.394 & 1.405 & 0.648 & 0.427 \\
\hline 296 & & & & $\mathrm{G}$ & 1.380 & 1.391 & 1.371 & 1.383 & 0.629 & 0.573 \\
\hline 297 & & & & $\mathrm{~J}$ & 1.480 & 1.523 & 1.513 & 1.490 & 0.420 & 0.045 \\
\hline 298 & & & & $\mathrm{~L}$ & 1.370 & 1.366 & 1.353 & 1.362 & 0.671 & 0.727 \\
\hline 299 & & & & $\bar{M}$ & 1.420 & 1.454 & 1.428 & 1.434 & 0.553 & 0.273 \\
\hline 300 & & & & $\mathrm{O}$ & 1.380 & 1.404 & 1.382 & 1.393 & 0.602 & 0.500 \\
\hline
\end{tabular}


A Comparative Study of Bond Order and Bond Length Calculations of Some Conjugated Hydrocarbons using Two Different Methods

\begin{tabular}{|l|l|l|l|l|l|l|l|l|l|l|}
\hline \hline 301 & & & & $\mathrm{E}$ & 1.390 & 1.391 & 1.371 & 1.383 & 0.688 & 0.573 \\
\hline 302 & & & & $\mathrm{D}$ & 1.390 & 1.419 & 1.394 & 1.405 & 0.580 & 0.427 \\
\hline 303 & & & & $\mathrm{~B}$ & 1.450 & 1.476 & 1.452 & 1.452 & 0.506 & 0.191 \\
\hline 304 & & & & $\mathrm{~A}$ & 1.350 & 1.354 & 1.345 & 1.353 & 0.774 & 0.809 \\
\hline 305 & & & & $\mathrm{~K}$ & 1.440 & 1.466 & 1.441 & 1.444 & 0.497 & 0.227 \\
\hline 306 & & & & $\mathrm{~N}$ & 1.420 & 1.466 & 1.441 & 1.444 & 0.468 & 0.227 \\
\hline 307 & & & & $\mathrm{H}$ & 1.440 & 1.428 & 1.403 & 1.413 & 0.540 & 0.382 \\
\hline 308 & & & & $\mathrm{C}$ & 1.420 & 1.428 & 1.403 & 1.413 & 0.536 & 0.382 \\
\hline 309 & & & & $\mathrm{I}$ & 1.420 & 1.464 & 1.438 & 1.442 & 0.504 & 0.236 \\
\hline
\end{tabular}

\section{Conclusions}

It is concluded that Pauling definition was important to predict the bond orders for Kekuléanbenzenoids. The predicted bond orders were positive and in range between zero for single bond, one for double bonds and fractions for intermediate bonds. And the Coulson definition was crucial to predict the bond orders for benzenoids and non benzenoids where the predicted bond orders were positive and negative fractions. The comparison between both definitions showed that Coulson bond order $\mathrm{CBO}$ was more bonds differentiated than Pauling bond order PBO since the definition of PBO depends on counting of Kekulé structures of a benzenoid but CBO is a sum of coefficients of atoms for each bond of molecule.

Two versions of Pauling equation that predicted in 1948 and 1960 were used to calculate the bond lengths. The two equations were successful and precise to predict the bond lengths. A deviation of predicted lengths $R_{1960}$ and $R_{1948}$ from experiment was about (0. 068) $\AA$ and (0.078) $\AA$, resectively. The linear correlations between measured bond lengths and the calculated $R$ in 1948 and 1960 were with $R^{2}$ equal to (0.7477) and (0.7783), respectively. The closeness between these equations was also investigated which obviously appeared through the linear correlation with $R^{2}$ equal to (0.982).

Pauling equation of bond length with new parameters, called $R_{\text {new }}$ was tested. These parameters were $\left(1.54 \AA\right.$ for single bond $R_{1}, 1.33 \AA$ for double bond $R_{2}$ and 1.84 for force constant $F$ ). As shown, a deviation of predicted lengths $R_{\text {new }}$ from experiment was about $(0.094) \AA$. The measured bond lengths $R_{\text {exp }}$ and the lengths that predicted using different versions of Pauling equation $\left(R_{1948}, R_{1960}\right.$ and $\left.R_{\text {new }}\right)$ were correlated. The correlation showed that the $R_{\text {new }}$ had as the same value of $R^{2}(0.7783)$ as $R_{1960}$.

The best value of the ratio of force constant in Pauling equation of bond length of the set of molecules was also determined. Different values of $F$ and 101 values of bond lengths range between (1.33-1.54) $\AA$ and 101 bond orders range between ( 0 and one) were used. The results have been shown that for the set of benzenoids, Pauling equation was fitting to experiment at $F$ equal to (2.5).

\section{REFERENCES}

[1] Pauling,L.; Brockway, L. and Beach,J.1935. The dependence of interatomic distance on single bond-double bond resonance. J. Am. Chem. Soc., 57 : 2705-2709.

[2] Lennard-Jones, J. E. 1937. The electronic structure of some polynes and aromatic molecules. I. The nature of the links by the method of molecular orbitals. Proc. R. Soc. Lond. A, 158: 280296.

[3] Penney, W. G.1937.The electronic structure of some polynes and aromatic molecules. III. Bonds of fractional orders by the pair method. Proc. R. Soc. Lond. A, 158: 306-324.

[4] Coulson,C. A. 1939.The electronic structure of some polynes and aromatic molecules. VII. Bonds of fractional orders by the molecular orbital method. Proc. R. Soc. Lond. A, 169: 413-428.

[5] Sedlar, J. ;Anđelić,I. ; Gutman,I. ; Vukičević, D. and Graovac,A. 2006. Vindicating the Paulingbond-order concept. Chem. Phys. Lett., $427:$ 418-420.

[6] Cao,Z.; Wu, W. and Zhang, Q. 2003. Bond length features of linear carbon chains of finite to infinite size : visual interpretation from Pauling bond order. Int. J. Quantum Chem., 94 : 144149.

[7] Cyvin, S. J. and Gutman,I.1987. A new method of enumeration ofKekulé structures. Chem. Phys. Lett., 136(2): 137-140.

[8] Cyvin,S. J. 1983. Number and symmetry of Kekulé structures for some aromatic chain molecules. Monatshefte für Chemie, 114: 13-19. 
[9] McWeeny,R. 1979. Coulson Valence, Oxford University Press, Oxford, Third Edit.

[10] Ham, N. S. and Ruedenberg, K. 1958. Mobile bond orders in conjugated systems. J. Chem. Phys., 29: 1215.

[11] Pauling, L. 1980. Bond numbers and bond lengths in tetrabenzo[de,no,st, $\left.\mathrm{c}_{1}, \mathrm{~d}_{1}\right]$ heptacene and other condensed aromatic hydrocarbons: a valence bond treatment. Acta Cryst., B36: 18981901.

[12] Herndon, W. C. 1976. П bond orders and bond lengths. Journal of Chemical Education. 53:689692.

[13] Kiralj,R. and Ferreira,M. M. C. 2002. Predicting bond lengths in planar benzenoid polycyclic aromatic hydrocarbons: a chemometric approach. j. Chem. Inf. Comput. Sci., 42: 508-523.

[14] Nyhoff, L. and Leestma, S. 1995. Fortran 77 and Numerical Methods for Engineers and Scientists, Prentic Hall, Englewood Cliffs, New Jersey

[15] Jenneskens, D.; Fowler,P. W.; Myrvold,W. and Bird, W. H. 2016. Perimeter ring currents in benzenoids from Pauling bond orders. J. Phys. Chem. Chem. Phys., 18(17): 11756-11764. 